\title{
SUMS OF LAURENT AND BILATERAL HANKEL OPERATORS
}

\author{
by G. J. MURPHY
}

(Received 23rd October 1986)

One can define "Laurent" and "Hankel" operators relative to groups more general than the circle group $\mathbb{T}$. We do that here, derive some of their properties, and compute their spectra, using a concrete realization of a crossed product $C^{*}$-algebra by the twoelement group $\mathbb{Z}_{\mathbf{2}}$.

Definition. Let $A$ be a $C^{*}$-algebra and $\gamma: A \rightarrow A$ a $*$-isomorphism such that $\gamma^{2}=\mathrm{id}_{A}$. We define

$$
C^{*}(A, \gamma)=\left\{\left(\begin{array}{cc}
a & b \\
\gamma b & \gamma a
\end{array}\right): a, b \in A\right\}
$$

It is readily verified that $C^{*}(A, \gamma)$ is a $C^{*}$-subalgebra of the $2 \times 2$ matrix algebra $M_{2}(A)=$ $M_{2} \otimes A$ over $A$.

Proposition 1. Let $B$ be a unital $C^{*}$-algebra and $A$ a $C^{*}$-subalgebra of $B$ containing the unit 1 of $B$. Let $s \in B$ be a symmetry (i.e. Hermitian unitary) such that $s A s=A$, and define the *-isomorphism $\gamma: A \rightarrow A$ by $a \mapsto$ sas.

Then $A+A s(=\{a+b s: a, b \in A\})$ is $a C^{*}$-subalgebra of $B$ and the map

$$
\phi: C^{*}(A \cdot \gamma) \rightarrow B,\left(\begin{array}{cc}
a & b \\
s b s & s a s
\end{array}\right) \mapsto a+b s,
$$

is a *-homomorphism with image $A+A s$.

Proof. Elementary computations show $\phi$ is a *-homomorphism, and its image is clearly $A+A s$, implying $A+A s$ is a $C^{*}$-subalgebra of $B$.

Corollary 2. $\phi$ is injective if $A \cap A s=0$.

Now suppose $G$ is a compact abelian group with dual group $\Gamma$. Define $S \in B\left(L^{2}(G)\right)$ by $S f=f$ where $f: x \rightarrow f(-x)$. $S$ is clearly a symmetry. If $\phi \in L^{\infty}(G)$ we define the Laurent operator $L_{\phi} \in B\left(L^{2}(G)\right)$ by $f \mapsto \phi f$, and the (bilateral) Hankel operator $B_{\phi}$ by $B_{\phi}=L_{\phi} S$.

$\Gamma$ forms an orthonormal basis for $L^{2}(G)$, and when we talk of the matrix of an operator $T \in B\left(L^{2}(G)\right)$ we shall mean relative to this basis. If $T$ has matrix $\left(a_{y, 8}\right)$ we say 
it is a Laurent (resp. Hankel) matrix if $a_{y+\rho, \delta+\rho}=a_{y, \delta}$ (resp. $a_{\gamma+\rho, \delta-\rho}=a_{y, \delta}$ ) for all $\gamma, \delta, \rho \in \Gamma$. Clearly Laurent operators have Laurent matrices and Hankel operators have Hankel matrices. The proof of the next proposition is the same as the classical case $(G=\mathbb{T})$ and is included for the sake of completeness.

Proposition 3. Let $G$ be a compact abelian group, and $T \in B\left(L^{2}(G)\right)$. If $T$ has Laurent (resp. Hankel) matrix then $T$ is a Laurent (resp. Hankel) operator.

Proof. Let $\Gamma=\hat{G}$ and $\langle\phi, \psi\rangle=\int_{G} \phi \psi$ if $\phi \bar{\psi} \in L^{1}(G)$. Let $\left(a_{y, \delta}\right)$ be the matrix of $T$. Suppose firstly this is a Laurent matrix and put $\phi=\sum_{\delta \in \Gamma} \alpha_{\delta, 0} \delta$ in $L^{2}(G)$. Let $\gamma \in \Gamma$, and $f \in L^{2}(G)$. Then

$$
\begin{aligned}
\langle\phi f, \gamma\rangle & =\left\langle f, \sum_{\delta \in \Gamma} \bar{a}_{\delta, 0} \delta \gamma\right\rangle \\
& =\sum_{\delta \in \Gamma} a_{\delta, 0}\langle f, \delta \gamma\rangle=\sum_{\delta \in \Gamma} \alpha_{\gamma-\delta, 0}\langle f, \delta\rangle \\
& =\sum_{\delta \in \Gamma} a_{\gamma, \delta}\langle f, \delta\rangle, \text { since } a_{\gamma-\delta, 0}=a_{\gamma, \delta}
\end{aligned}
$$

Thus $\langle\phi f, \gamma\rangle=\langle T f, \gamma\rangle(\gamma \in \Gamma)$, implying $\phi f=T f$. Hence $\phi L^{2}(G) \subseteq L^{2}(G)$ and so $\phi \in L^{\infty}(G)$. Thus $T=L_{\phi}$ is a Laurent operator.

On the other hand, if $T$ has Hankel matrix then $T S$ has Laurent matrix so $T S=L_{\phi}$ i.e. $T=B_{\phi}, \exists \phi \in L^{\infty}(G)$.

Remark. It is easy to calculate the spectra for $L_{\phi}$ and $B_{\phi}$. The map $L^{\infty}(G) \rightarrow B\left(L^{2}(G)\right)$, $\phi \rightarrow L_{\phi}$, is clearly an isometric $*$-homomorphism, so $\left\|L_{\phi}\right\|=\|\phi\|_{\infty}$ and $\sigma\left(L_{\phi}\right)=\sigma(\phi)$ (the spectrum relative to $L^{\infty}(G)$ ). Also $B_{\phi}^{2}=L_{\phi}\left(S L_{\phi} S\right)=L_{\phi} L_{\phi}=L_{\phi \phi}$, so $\sigma\left(B_{\phi}\right)^{2}=\sigma(\phi \not)$ ). We need to do a little more to calculate $\sigma\left(L_{\sigma}+B_{\psi}\right)$ for $\phi, \psi \in L^{\infty}(G)$ :

Proposition 4. If $G$ is a nontrivial connected compact abelian group, then $C=$ $\left\{L_{\phi}+B_{\psi}: \phi \psi \in L^{\infty}(G)\right\}$ is a $C^{*}$-subalgebra of $B\left(L^{2}(G)\right)$. If $\phi, \psi \in L^{\infty}(G)$ then

$$
\begin{aligned}
\max \left\{\|\phi\|_{\infty},\|\psi\|_{\infty}\right\} \leqq\left\|L_{\phi}+B_{\psi}\right\| \leqq 2 \max \left\{\|\phi\|_{\infty},\|\psi\|_{\infty}\right\} \text { and } \\
\sigma\left(L_{\phi}+B_{\psi}\right)=\left\{\lambda \in \mathbb{C}:(\lambda-\phi)(\lambda-\phi)-\psi \psi \text { is noninvertible in } L^{\infty}(G)\right\} .
\end{aligned}
$$

Proof. We can replace the condition $G$ is connected by the weaker one that $\Gamma=\hat{G}$ has an element of infinite order. (That $G$ connected implies this condition on $\Gamma$ is an elementary exercise.)

Let $A=\left\{L_{\phi}: \phi \in L^{\infty}(G)\right\}$. If $T \in A \cap A S$ and has matrix $\left(a_{y, \delta}\right)$ and $\rho$ is an element of infinite order in $\Gamma$ then $(\gamma+2 n \rho, \delta)_{n=1}^{\infty}$ is an infinite sequence in $\Gamma \times \Gamma$ and since $a_{y, \delta}=a_{y+\rho, \delta+\rho}=a_{y+2 \rho, \delta}$, so $a_{y, \delta}=a_{y+2 n \rho, \delta}(n=1,2,3, \ldots)$. We have $\sum_{n=1}^{\infty}\left|a_{y+2 n \rho, \delta}\right|^{2}<\infty$, and so $a_{y, \delta}=0$. Thus $A \cap A S=0$. 
We now deduce from Proposition 1 and Corollary 2 that the map

$$
C^{*}(A, \psi) \rightarrow A+A S,\left(\begin{array}{ll}
L_{\phi} & L_{\psi} \\
L_{\psi} & L_{\phi}
\end{array}\right) \mapsto L_{\phi}+B_{\psi}
$$

is a *-isomorphism onto the $C^{*}$-subalgebra $C=A+A S$ of $B\left(L^{2}(G)\right)(\mu: A \rightarrow A$ is defined by $\left.L_{\phi} \rightarrow L_{\phi}\right)$.

Let $\phi, \psi \in L^{\infty}(G)$. Clearly

$$
\left\|L_{\phi}+B_{\psi}\right\|=\left\|\left(\begin{array}{ll}
L_{\phi} & L_{\psi} \\
L_{\psi} & L_{\phi}
\end{array}\right)\right\| \geqq\left\|L_{\phi}\right\|=\|\phi\|_{\infty}
$$

and since

$$
\left\|L_{\phi} S+B_{\psi} S\right\|=\left\|L_{\psi}+B_{\phi}\right\| \geqq\|\psi\|_{\infty}
$$

we have

$$
\left\|L_{\phi}+B_{\psi}\right\| \geqq \max \left(\|\phi\|_{\infty},\|\psi\|_{\infty}\right)
$$

Also

$$
\left\|L_{\phi}+B_{\psi}\right\| \leqq\|\phi\|_{\infty}+\|\psi\|_{\infty} \leqq 2 \max \left\{\|\phi\|_{\infty},\|\psi\|_{\infty}\right\} .
$$

Finally $L_{\phi}+B_{\psi}-\lambda$ is invertible iff

$$
\left(\begin{array}{ll}
L_{\phi-\lambda} & L_{\psi} \\
L_{\psi} & L_{\phi-\lambda}
\end{array}\right)
$$

is invertible iff the determinant $L_{\phi-\lambda} L_{\phi-\lambda}-L_{\psi \psi}$ is invertible. (This is an elementary computation which works because all the matrix entries commute.) Thus $L_{\phi}+B_{\psi}-\lambda$ is invertible iff $(\phi-\lambda)(\phi-\lambda)-\psi \psi$ is invertible in $L^{\infty}(G)$.

Remark. The results of Proposition 4 were obtained by Walsh ([4], unpublished) in the case of $G=\mathbb{T}$ by methods quite different from ours. He analyses these operators in greater detail in this classical case.

Corollary 5. If $\phi, \psi \in L^{\infty}(G)$ and $\phi(x)=\phi(-x), \psi(x)=\psi(-x)(x \in G)$ then $\sigma\left(L_{\phi}+B_{\psi}\right)=$ $\sigma(\phi+\psi) \cup \sigma(\phi-\psi)$.

Proof. In this case $(\lambda-\phi)(\lambda-\phi)-\psi \psi$

$$
=(\lambda-\phi)^{2}-\psi^{2}=(\lambda-\phi-\psi)(\lambda-\phi+\psi) .
$$


These results are not true for arbitrary $G$. If $G$ is the Cantor group $\left(\mathbb{Z}_{2}\right)^{\mathrm{N}}$, then $x=-x$ ( $x \in G$ ), implying $S=$ id and so $A \cap A S \neq 0$. Hence $\sigma\left(L_{\phi}+B_{\psi}\right)=\sigma(\phi+\psi) \neq \sigma(\phi+\psi) \cup \sigma(\phi-\psi)$ in general (Take $\phi=\psi$ invertible).

Here is a trivial but interesting remark:

Take $G=\mathbb{T}$ and $E$ any subset of $\mathbb{T}$ of positive measure not intersecting $E^{-1}=$ $\left\{x^{-1}: x \in E\right\}$. Let $\phi=x_{E}$. Then $\phi \in L^{\infty}(\bar{T}), \phi \Phi=0$, and so $B_{\phi}^{2}=L_{\phi \phi}=0$, but $B_{\phi} \neq 0$.

This is quite different from the behaviour of unilateral Hankel operators on the Hardy space $H^{2}$, none of which (0 excluded) are nilpotent. (See [3], [4]).

Now let us take a brief closer look at the $C^{*}$-algebra $C^{*}(A, \gamma)$ where $A$ is a unital $C^{*}$ algebra and $\gamma: A \rightarrow A$ is a *-isomorphism such that $\gamma^{2}=$ id. Define an action of $\mathbb{Z}_{2}$ on $A$ by $\tilde{\gamma}: \mathbb{Z}_{2} \rightarrow$ Aut $A, \tilde{\gamma}(0)=\mathrm{id}, \tilde{\gamma}(1)=\gamma$. Then the crossed product [1] $B=A x_{\tilde{\gamma}} \mathbb{Z}_{2}$ has a symmetry $s$ such that $s A s=A, B=A+A s$, and $A$ contains the unit of $B$. Moreover $A \cap A s=0$. Thus we have a *-isomorphism

$$
C^{*}(A, \gamma) \rightarrow A x_{\tilde{\gamma}} \mathbb{Z}_{2},\left(\begin{array}{cc}
a & b \\
\gamma b & \gamma a
\end{array}\right) \rightarrow a+b s
$$

(This follows from Proposition 1 and Corollary 2.)

In particular, if $G$ is a connected compact abelian group then the $C^{*}$-algebra $\left\{L_{\phi}+B_{\psi}\right.$ : $\left.\phi, \psi \in L^{\infty}(G)\right\}$ is just the crossed product $L^{\infty}(G) x_{\mu} \mathbb{Z}_{2}$ for a suitable action $\mu$.

A final remark. In the study of $C^{*}$-algebras of the related class of Toeplitz operators, much use is made of commutator ideals and their identification. That this approach is not appropriate in the context of the algebra $C=\left\{L_{\phi}+B_{\psi}: \phi, \psi \in L^{\infty}(G)\right\}$ is indicated by the following. Suppose there exists a Borel set $E$ in $G$ with $-E=G \backslash E$. (This is obviously true for $G=\mathbb{T}$.) Let $\phi=x_{E}$. Then $\phi=x_{-E}=1-x_{E}=1-\phi$. Let $\psi=2 \phi-1$. Then $\psi^{2}=1$. But

$$
\psi=\frac{\psi-\psi}{2} \quad(\text { since } \psi=2 \phi-1=2(1-\phi)-1=1-2 \phi=\psi)
$$

and so

$$
L_{\psi}=\frac{1}{2}\left(L_{\psi}-S L_{\psi} S\right)=\frac{1}{2}\left(L_{\psi} S-S L_{\psi}\right) S .
$$

Thus if $I$ is the commutator ideal of $C$ (i.e. the smallest closed ideal containing all commutators $x y-y x, x, y \in C)$ then $L_{\psi} \in I$ and so $1=L_{\psi}^{2} \in I$. Thus $I=C$.

Acknowledgement. I would like to thank the referee for bringing to my attention a paper of S. C. Power [2] to which the context of this paper is related. In particular the following result is shown there (cf. Proposition 1 of this paper): If $A$ is a $C^{*}$-subalgebra of a $C^{*}$-algebra $B$ and $M$ is a closed $A$ bi-module of $B$ with $M=M^{*}$ and $M^{2} \subseteq A$ then $A+M$ is a $C^{*}$-algebra. 


\section{REFERENCES}

1. G. Pedersen, $C^{*}$-algebras and their Automorphism Groups (Academic Press, London, 1979).

2. S. C. Power, $C^{*}$-modules and an odd even decomposition for $C^{*}$-algebras, Bull. London Math. Soc. 8 (1976), 268-272.

3. S. C. Power, Quasinilpotent Hankel operators, in Linear and Complex Analysis Problem Book (V. P. Havin, S. V. Hrusćev and N. K. Nikolskii (editors), Springer Lecture Notes, 1043, Springer, 1984).

4. D. WALSH, Thesis (University College Cork, 1969), unpublished.

Department of Mathematics

University College, Cork

IRELAND 